\title{
Immunogenicity of envelope GP5 protein displayed on baculovirus and protective efficacy against virulent porcine reproductive and respiratory syndrome virus challenge in piglets
}

\author{
Zhisheng Wang ${ }^{1 \mathrm{a}}$, Xingang $\mathrm{Xu}^{1 \mathrm{a}}$, Hungjen $\mathrm{Liu}^{2}$, Zhaocai $\mathrm{Li}^{1}$, Li Ding ${ }^{1}$, Gaoshui $\mathrm{Yu}^{1}$, Dan Xu${ }^{1}$ and Dewen Tong ${ }^{1 *}$ \\ ${ }^{1}$ College of Veterinary Medicine, Northwest A and F University, Yangling, Shaanxi 712100, P.R. China. \\ ${ }^{2}$ Institute of Molecular Biology, National Chung Hsing University, Taichung 402, Taiwan.
}

Accepted 30 July, 2011

\begin{abstract}
In the present study, one recombinant baculovirus BacSC-GP5, expressing His6-tagged GP5 with the transmembrane domain (TM) and cytoplasmic domain (CTD) derived from baculovirus envelope protein gp64, was constructed and its immunogenicity and protective efficiency was evaluated in piglets. The results obtained show that, His6-tagged recombinant GP5 was expressed and anchored on the plasma membrane of Sf-9 cells, as revealed by Western blot and confocal microscopy. Immunogold electron microscopy demonstrated that, the GP5 glycoprotein was displayed successfully on the viral surface. Piglets immunized with BacSC-GP5 induced successfully GP5-specific enzyme-linked immunosorbent assay (ELISA) antibody, neutralizing antibody and lymphocyte proliferation response at 6 weeks after primary immunization. An in vivo challenge result indicated that piglets immunized with BacSC-GP5 did not show any obvious clinical signs and histological changes, and the quantitative real-time polymerase chain reaction (RT-PCR) also indicated that the porcine reproductive and respiratory syndrome virus (PRRSV) viral load from the serum in BacSC-GP5 group was significantly reduced at 14 and 21 days post-challenge compared to that in the negative control group. These results indicate that baculovirus-mediated gene delivery can be utilized as an alternative strategy to develop a new generation of vaccine against PRRSV infection.
\end{abstract}

Key words: Baculovirus surface display, porcine reproductive and respiratory syndrome virus (PRRSV), GP5 protein, immunogenicity, protective efficacy.

\section{INTRODUCTION}

Porcine reproductive and respiratory syndrome (PRRS), characterized by severe reproductive failure in late term gestation in sows and respiratory disease in pigs of all ages, is considered as one of the most economically important diseases affecting the swine industry worldwide (Meulenberg, 2000). PRRSV, the causative agent, is an envelope, positive stranded ribonucleic acid (RNA) virus

${ }^{*}$ Corresponding author. E-mail: dwtong@nwsuaf.edu.cn. Tel: 0086-29-8709-1622. Fax: 0086-29-8709-1032.

\footnotetext{
a Both two authors contributed equally to this work
}

belonging to the family Arteriviridae (www.ictvdb.org/lctv/index.htm). The viral genome is approximately $15 \mathrm{~kb}$ in length and contains nine open reading frames (ORF1a/1b, ORF2a/2b, ORF3-7) (Meulenberg et al., 1995). The GP5, encoded by ORF5 gene, is one of the key immunogenic proteins of PRRSV involved in the generation of PRRSV-neutralizing antibody and protective immunity and is one of the leading targets for the development of the genetic engineering vaccines against PRRS (Barfoed et al., 2004; Zheng et al., 2007). Several experimental vaccines expressing GP5 protein have been studied and tested in recent years, such as recombinant fowlpox virus (Shen et al., 2007), 


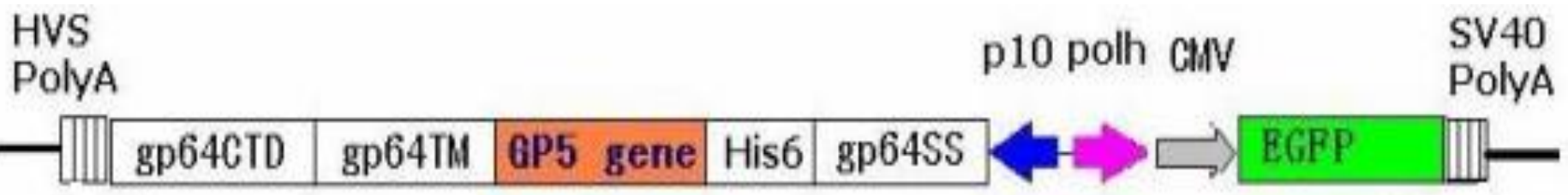

Figure 1. Schematic diagram of the recombinant baculovirus vector pBacSC-GP5. Plasmid pBacSC was constructed using pFastBac Dual (Invitrogen, Carlsbad, USA) as the backbone. The fusion gene fragments of baculovirus gp64 SS, His6, multiple cloning sites (Xho I, Xba I, Pst I, and EcoRI), baculovirus gp64 TM, baculovirus gp64 CTD were inserted into the corresponding site under the control of baculovirus p10 promoter. The immediate early promoter of human cytomegalovirus (CMV) and the enhanced green fluorescent protein (EGFP) coding sequences were cloned into the corresponding site under the control of baculovirus polyhedrin promoter (Ppolh). The GP5 gene was inserted into the pBacSC vector. The resultant plasmid was named as pBacSC-GP5.

recombinant adenovirus (Jiang et al., 2006a), and recombinant pseudorabies virus vaccines (Jiang et al., 2007; Qiu et al., 2005). Although partial protective immunity can be obtained with these experimental vaccines, one major problem in using these recombinant viruses as vaccines is the pathogenic potential of the vector virus.

Baculovirus has captured increasing interest as a vector due to its safety and abundant yields. Several reports have demonstrated that baculovirus carrying mammalian cell-active promoters is capable of transferring and expressing foreign genes in a variety of mammalian cells, as well as in animal models (Tani et al., 2003). Furthermore, it has also been reported that a pseudotyped baculovirus displaying the glycoprotein of vesicular stomatitis virus (VSV-G) on the envelope can extend the host range and increase the efficiency of transduction in mammalian cells (Pieroni et al., 2001). In recent years, several researchers have developed a baculovirus surface display vector, utilizing the baculovirus envelope glycoprotein-gp64 which is comprised of an N-terminal signal sequence and a mature domain including the transmembrane domain (TM) and cytoplasmic domain (CTD) (Kitagawa et al., 2005). And the heterologous gene was inserted between the SS and the mature domain. This method has been extended to develop various pseudotyped baculoviruses as potential vaccines, such as expressing avian influenza virus (AIV) hemagglutinin protein (Yang et al., 2007) and classic swine fever virus (CSFV) E2 protein (Xu and Liu, 2008). These researchers have demonstrated that direct vaccination with recombinant baculovirus could induce high-level humoral and cell-mediated immunity in mouse model or natural host.

The main objective of this study is to use the baculovirus surface display system expressing the PRRSV GP5 protein on the baculoviral envelope, in the hope that GP5 would retain superior immunogenicity upon in vivo immunization. Here, we constructed a baculovirus surface display vector expressing the GP5 protein on the baculoviral envelope and evaluated its immunogenicity and protective efficacy in piglets.

\section{MATERIALS AND METHODS}

\section{Cells and viruses}

The Spodoptera frugiperda 9 (Sf-9) cell lines were grown at $27^{\circ} \mathrm{C}$ in Grace's insect media (Invitrogen, Carlsbad, USA) supplemented with $10 \% \quad(\mathrm{v} / \mathrm{v})$ heat-inactivated fetal bovine serum (FBS) (Gibco-BRL, Carlsbad, USA), $100 \mathrm{U} / \mathrm{ml}$ of penicillin and $100 \mu \mathrm{g} / \mathrm{ml}$ of streptomycin. The PRRSV Shaanxi strain (Wang et al., 2010), a highly pathogenic North American type isolate, was used in this study. PRRSV was propagated in MARC-145 cells, which were grown and maintained in Dulbecco's modified Eagle's medium (DMEM) (Invitrogen, Carlsbad, USA) supplemented with 10\% FBS, $100 \mathrm{U} / \mathrm{ml}$ of penicillin and $100 \mu \mathrm{g} / \mathrm{ml}$ of streptomycin at $37^{\circ} \mathrm{C}$ with $5 \%$ $\mathrm{CO}_{2}$.

\section{Recombinant baculovirus construction and preparation}

The baculovirus surface display system, pBacSC plasmid, was constructed and provided by collaborators (Xu and Liu, 2008). To generate a recombinant baculovirus expressing the GP5 protein (BacSC-GP5), the DNA fragment of ORF5 (deleting the N-terminal signal peptide of 31 amino acid residues) was amplified from the plasmid pGEM-GP5 (containing the complete cDNA of ORF5 gene of the PRRSV Shaanxi strain, kept in our laboratory) by polymerase chain reaction (PCR) with forward primer (PRRSV-Xhol), 5'-GCTCTCGAGAGCAACAACAGCAG-3', and reverse primer (PRRSV-Pstl), 5'-GATCTGCAGGAGACGACCCCATTGTT-3'. The restriction sites are underlined. The amplified product was subcloned into the corresponding site in the pBacSC plasmid, resulting in the recombinant transfer plasmid pBacSC-GP5 (Figure 1). The recombinant baculovirus was subsequently generated by using the Bac-to-Bac ${ }^{\circledR}$ system (Invitrogen, Carlsbad, USA) following the manufacturer's instructions. Recombinant baculovirus was further amplified by propagation in Sf-9 cells. Virus purification was performed as described previously (Abe et al., 2004), and the titer was determined by a plaque assay according to the manufacturer's manual (Invitrogen, Carlsbad, USA).

\section{SDS-PAGE and Western blot analysis}

The infected cell lysates were subjected to $12 \%$ sodium dodecyl sulfate polyacrylamide gel electrophoresis (SDS-PAGE) and blotted to polyvinylidene fluoride (PVDF) membranes (Amersham, New York, USA). Two primary antibodies were used to detect GP5 in the Western blot: mouse monoclonal His-tag antibody (1:3000 dilution, 
Invitrogen, Carlsbad, USA) and porcine polyclonal PRRSV antibody (1:500 dilution, provided kindly by the Center of Animal Health and Epidemiology, Qingdao, China). The secondary antibodies were goat anti-mouse and rabbit anti-porcine IgG conjugated to HRP (1:5000 dilution, Invitrogen, Carlsbad, USA). The protein bands were visualized by the electrochemiluminescence (ECL) kit (Pierce, Rockford, USA). The purified virus particles were also detected by SDS-PAGE and Western blot analysis following the same protocol.

\section{Confocal microscopy}

The confocal microscopy was carried out as described previously (Yang et al., 2007). The cells were incubated with the primary antibodies (mouse monoclonal His-tag antibody and porcine polyclonal PRRSV antibody, 1:300 dilution) and the secondary antibodies (fluoresceine isothiocyanate (FITC)-conjugated goat anti-mouse and rabbit anti-porcine IgG, 1:1000 dilution, Invitrogen, Carlsbad, USA). The negative control cells were treated in the same way. Protein localization was visualized using a confocal microscope LSM 510 META (Zeiss, Berlin, Germany).

\section{Immunogold electron microscopy}

The immunogold electron microscopy was performed as described previously (Hu et al., 2003) with minor modifications. The grids were incubated with the porcine polyclonal PRRSV antibody (1:300 dilution) and the rabbit anti-porcine IgG conjugated with 10-nm gold particles (1:100 dilution, Sigma, St. Louis, USA). The grids were then examined under the transmission electron microscope $\mathrm{H}-7500$ (Hitachi, Tokyo, Japan).

\section{Immunization of piglets}

To evaluate whether the pseudotyped baculovirus BacSC-GP5 can elicit immune responses, fifteen healthy 4-week-old piglets were obtained from a PRRS-free farm in Xi'an, China. The piglets were tested seronegative to PRRSV by ELISA (IDEXX, New York, USA). The animals were then separated randomly into three groups (five piglets each group), numbered, and housed in separate rooms. One group was inoculated intramuscularly twice at 2-week internals with $1 \mathrm{ml}$ phosphate-buffered saline (PBS) containing $1.0 \times 10^{9}$ plaque forming units (PFU) of purified BacSC-GP5. The other two groups were injected respectively with $1 \mathrm{ml} \mathrm{PBS}$ containing $1.0 \times 10^{9} \mathrm{PFU}$ of purified BacSC or PBS as the negative control groups. Serum samples were collected from the $V$. jugularis external at 6 weeks after primary immunization for serological tests. Peripheral blood mononuclear cells (PBMCs) were isolated from whole blood with sodium heparin as an anticoagulant at 6 weeks after the primary immunization for lymphocyte proliferation assay.

\section{Serological tests}

GP5-specific antibody titers were tested using an indirect ELISA, with purified PRRSV as the coating antigen (Jiang et al., 2006a). The results were expressed as the ratio of OD490 produced by the serum samples compared with negative control serum. Sera with a ratio value higher than 2.1 were considered to be positive sera (Fan et al., 2008). The titers were expressed as the reciprocal of the highest dilution of sera producing ratio values of 2.1. Serum neutralization assays were performed as described previously (Jiang et al., 2006b). The neutralization titers were expressed as the reciprocal of the highest serum dilution in which no cytopathic effect (CPE) was observed. Each sample was run in quadruplicate.

\section{Lymphocyte proliferation assay}

Piglet PBMCs were isolated from the whole blood of immunized piglets with lymphocyte separation medium (TBD, Shanghai, China). The lymphocyte proliferation assay was performed as described previously (Jiang et al., 2006b). The stimulation index (SI) was calculated as the ratio of the average OD490 value of triplicate wells containing PRRSV stimulated cells to the average OD490 value of triplicate wells containing only cells with medium.

\section{Challenge and histopathological analysis}

At 6 weeks after primary immunization, all piglets were challenged intranasally with $1 \times 10^{5} 50 \%$ tissue culture infection dose $\left(T_{C I D}\right)$ of the PRRSV Shaanxi strain in $5 \mathrm{ml}$ of PRRSV-infected-MARC145 culture supernatant fluid. After challenge, the animals were monitored daily for the presence of clinical signs, including inappetence, depression, cough, dyspnoea, and fever. Rectal temperatures were recorded from 2 days pre-challenge to 21 days post-challenge (dpc). At $21 \mathrm{dpc}$, all piglets were dissected and macroscopic lesions were examined among the different groups. Histological changes were further evaluated in the collected lungs from the different groups. After fixation with $10 \%$ formalin, the lungs were embedded in paraffin, sectioned, and stained with hematoxylin and eosin (HE).

\section{Real-time RT-PCR analysis of viral replication in serum}

After challenge, serum samples were collected at 7, 14 and $21 \mathrm{dpc}$. The viral copy numbers of serum samples were estimated by $I Q^{\mathrm{TM}} 5$ real-time PCR system (Bio-Rad, Hercules, USA) using IQ ${ }^{\text {TM }}$ SYBR $^{\circledR}$ Green qPCR Kit (Bio-Rad, Hercules, USA). Briefly, the primers Q-PRRSV-F 5'-CAAATAACAACGGCAAGCAG-3' and Q-PRRSV-R 5'-AAACTCCACAGCGTAACTTA-3' were used to amplify a $308 \mathrm{bp}$ fragment from the ORF7 gene of PRRSV. The purified PCR product of PRRSV was cloned into pGEM-T easy vector and quantified by measuring $\mathrm{OD}_{260}$ using spectrophotometer (NanoDrop 2000, Wilmington, USA). A standard curve was obtained using serially diluted plasmid pGEM-ORF7 standards of $10^{1} \sim 10^{8}$ copies $/ \mu \mathrm{l}$ and was used to quantify the PRRSV viral genomic copy numbers. Viral RNA was extracted from $500 \mu$ of serum sample by using the MiniBEST Viral RNA Extraction Kit (Takara, Dalian, China). Then, PRRSV cDNA synthesis was performed according to the PrimeScript $^{\text {TM }}$ RT-PCR Kit (Takara, Dalian, China) with slight modification using $5 \mu \mathrm{l}$ of extracted RNA. The PCR amplification was carried out in a $25 \mu \mathrm{l}$ reaction volume containing $12.5 \mu \mathrm{I} \mathrm{IQ}^{\mathrm{TM}}$ SYBR $^{\circledR}$ Green supermix, 6 pM concentration of forward and reverse primers and $4 \mu \mathrm{l}$ cDNA. All reactions were carried out in triplicate. Statistical analysis was performed by using the means of three replicate wells for each sample as compared with a standard curve. PCR data was presented as follows: mean log10 genomic copies $/ \mathrm{ml}$ of serum \pm standard deviation.

\section{Statistical analysis}

Student's t-test was used to evaluate the differences among groups in regard to humoral and cellular immune responses, body temperatures and viral copy numbers. P-values $<0.05$ were considered statistically significant. 


\section{Anti-His6}

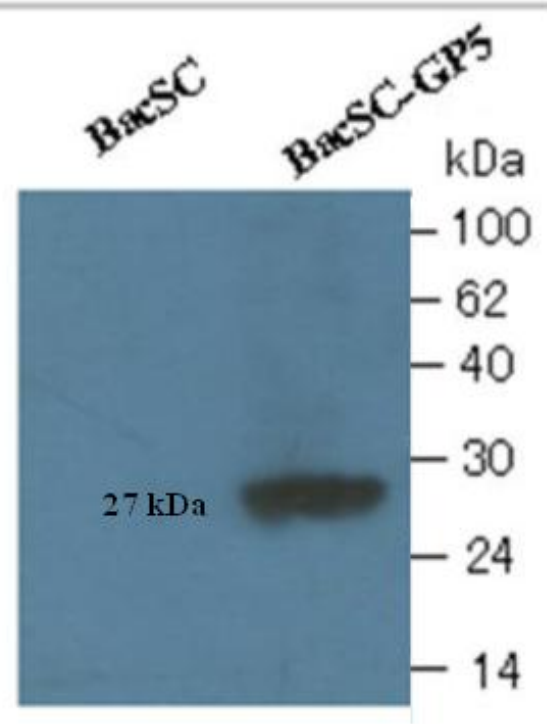

Anti-PRRSV

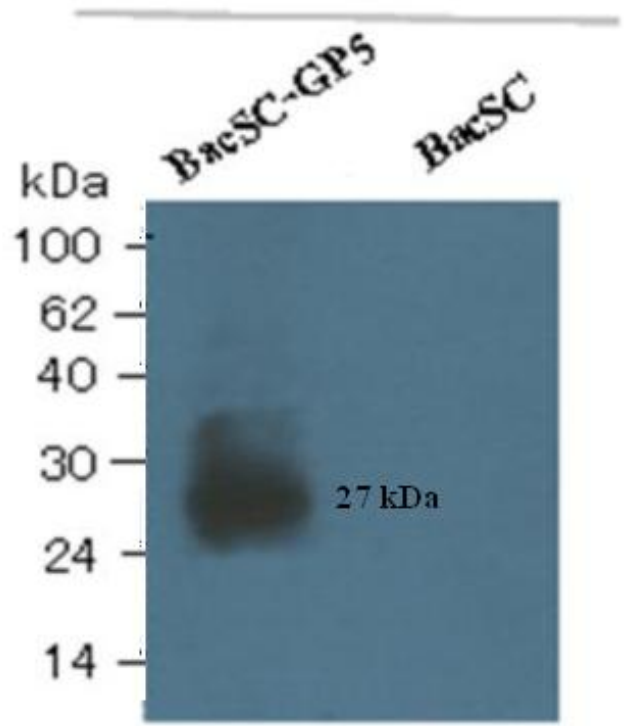

Figure 2. Western blot analysis of GP5 expression in insect cells. The Sf-9 cells were infected with BacSC-GP5 or BacSC at $10 \mathrm{MOI}$, harvested 3 days after infection, and subjected to Western blot assay. The $27 \mathrm{kDa}$ fusion proteins ( $23 \mathrm{kDa}$ of GP5 protein and $4 \mathrm{kDa}$ of His 6 fusion protein) were detected by His-tag and PRRSV antibodies. No proteins were detectable in the negative control (BacSC).

\section{RESULTS}

\section{GP5 expression from recombinant baculovirus in insect cells}

The Western blot analysis (Figure 2) showed that the cells infected by BacSC-GP5 expressed an approximately molecular mass of $27 \mathrm{kDa}$ fusion proteins (23 kDa of GP5 protein and $4 \mathrm{kDa}$ of His6 fusion protein) that were detected by both His-tag and PRRSV antibodies. This is in agreement with the predicted size from the GP5 protein. In contrast, there were no proteins recognized by His-tag and PRRSV antibodies in the BacSC.

The confocal microscopy result (Figure 3) revealed that there were no proteins recognized by His-tag and PRRSV antibodies in the negative control (Sf-9 cells only). In contrast, the GP5 protein could be detected by the His-tag and PRRSV antibodies in the BacSC-GP5 infected cells, whereas in the BacSC infected cells it could only be detected by the His-tag antibody. The protein localizes with the plasma membrane, thus demonstrating the anchoring of GP5 on the surface of Sf-9 cells.

\section{Display of GP5 on recombinant baculovirus}

To investigate whether the His6-tagged GP5 is incorporated into baculovirus, BacSC-GP5 and BacSC were purified by sucrose gradient ultracentrifugation and then subjected to SDS-PAGE and Western blot analysis. Figure 4 revealed that there was no target protein expression in the BacSC. In contrast, the protein expressed by BacSC-GP5 was detected by both His-tag and PRRSV antibodies. The purified viral particles were further analyzed by immunogold electron microscopy using porcine PRRSV primary antibody and anti-porcine IgG secondary antibody conjugated with $10-\mathrm{nm}$ gold particles. Figure 5 revealed that gold particles displayed on the surface of BacSC-GP5 (A) but not on the surface of BacSC (B), indicating the GP5 was incorporated and displayed on the baculoviral envelope. Moreover, the incorporation of GP5 did not alter the virus morphology.

\section{GP5-specific humoral and cellular immune responses in piglets}

As shown in Table 1, piglets vaccinated with BacSC-GP5 produced ELISA antibody with titers of 1:83.2 and neutralizing antibody with titers of 1:11.2. No GP5 specific ELISA and neutralizing antibodies could be detected in piglets immunized with BacSC or PBS throughout this experiment.

At 6 weeks after primary immunization, PBMCs were isolated and restimulated in vitro with the purified PRRSV $(20 \mu \mathrm{g} / \mathrm{ml})$ to analyze the cellular immune responses. As shown in Table 1, significantly higher lymphocyte proliferative responses could be observed in piglets 


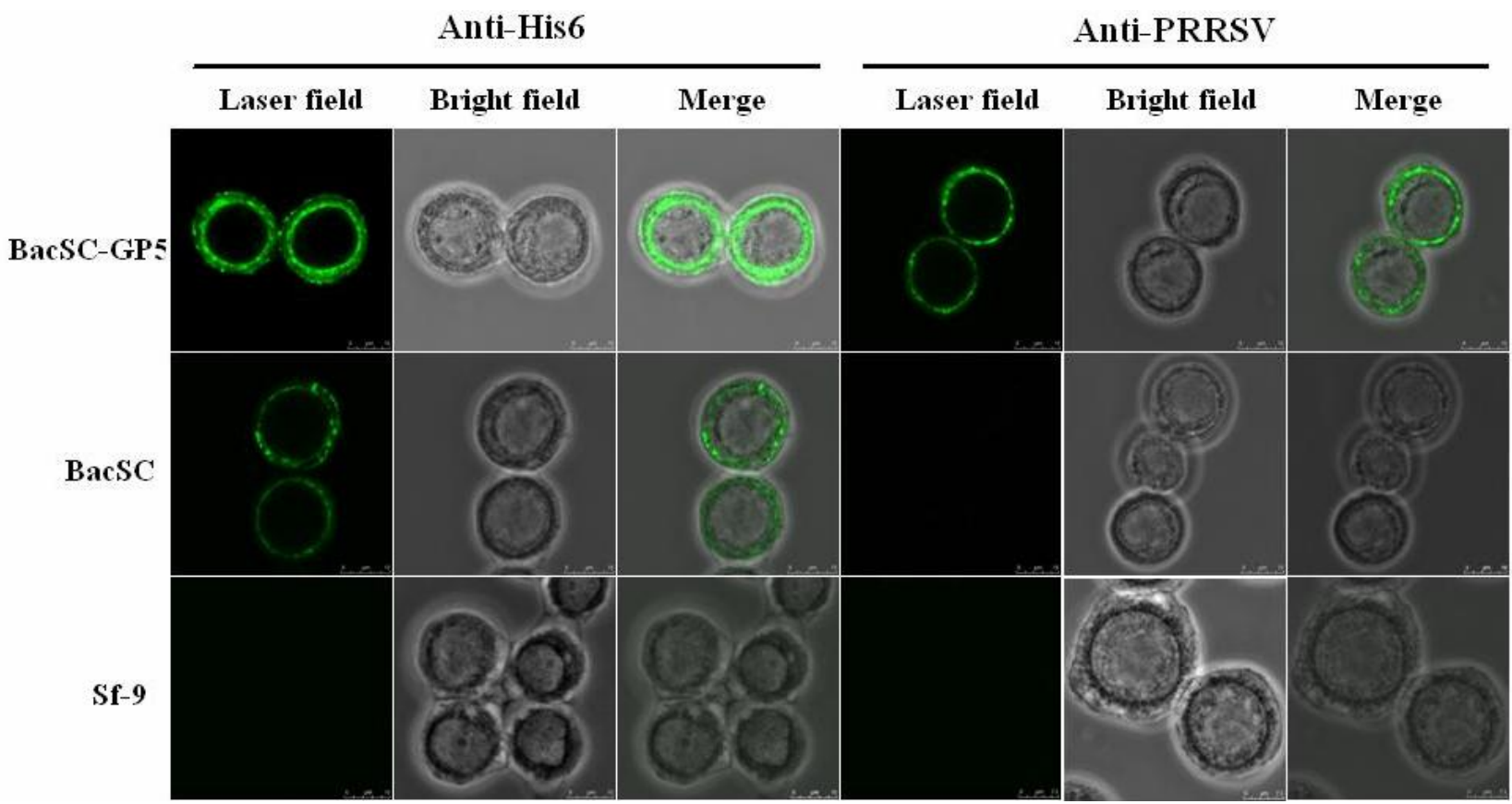

Figure 3. Confocal immunofluorescence microscopy of GP5 expression in insect cells. The cells were cultured on sterile cover slips, infected separately with BacSC-GP5 or BacSC at $10 \mathrm{MOI}$, and subjected to immunofluorescence examination by confocal microscopy 2 days after infection. The result showed that GP5 was translocated properly to the plasma membrane of the infected Sf-9 cells.

Anti-His6

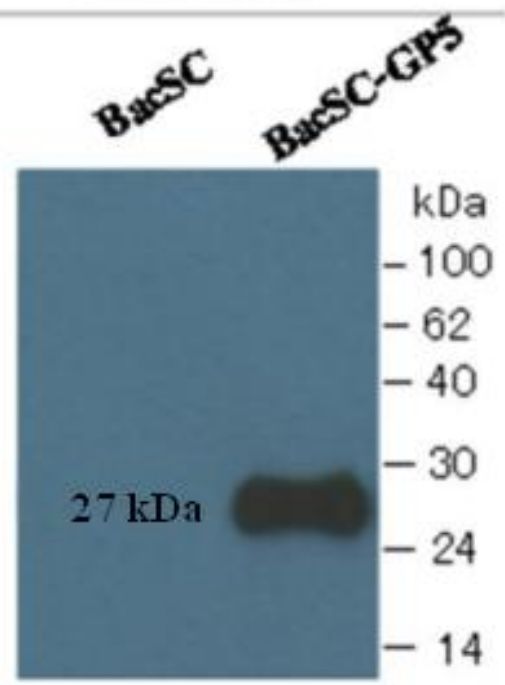

Anti-PRRSV

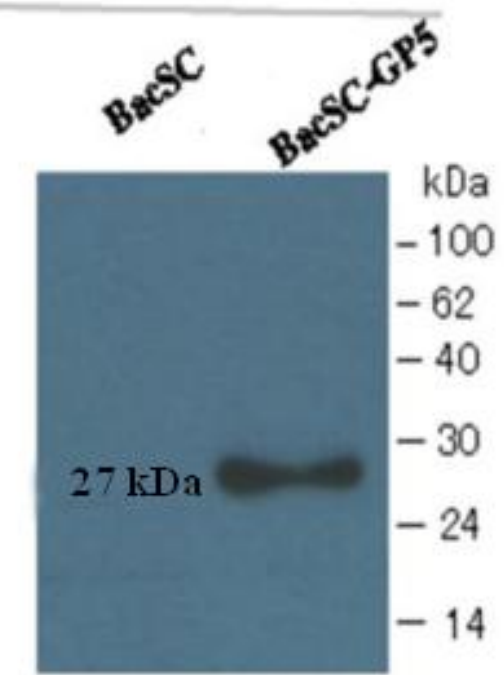

Figure 4. Western blot analysis of recombinant baculovirus. The budded viruses produced by infection of Sf-9 cells with recombinant viruses BacSC-GP5 or BacSC were purified by ultracentrifugation on sucrose gradient. The samples were subjected to Western blot assay and the GP5 fusion proteins $(27 \mathrm{kDa}$ ) were detected by His-tag monoclonal antibody and PRRSV polyclonal antibody. No proteins were detectable in the negative control (BacSC).

immunized with BacSC-GP5 compared to those with BacSC or PBS $(P<0.05)$. These data indicate that the pseudotyped baculovirus BacSC-GP5 can induce humoral and cellular immune responses in the natural 

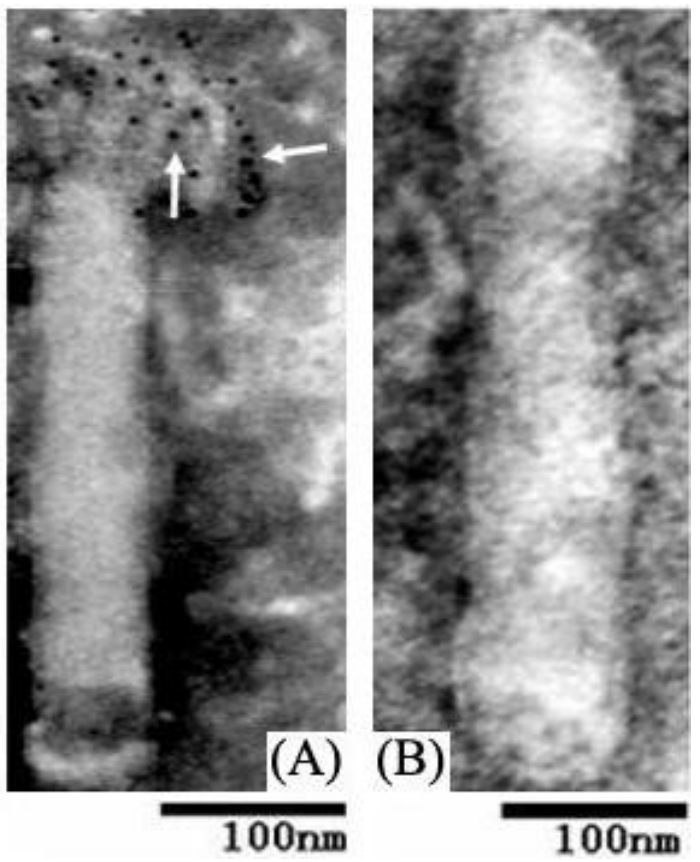

Figure 5. Incorporation of GP5 into the baculoviral envelope. Immunogold electron micrographs of purified baculovirus BacSC-GP5(A) or BacSC(B) detected by PRRSV polyclonal antibody as the primary antibody and anti-porcine IgG conjugated with $10-\mathrm{nm}$ gold particles as the secondary antibody. No gold particles were displayed on the surface of baculovirus BacSC.

Table 1. Humoral and cellular immune responses of piglets to recombinant baculovirus.

\begin{tabular}{|c|c|c|c|}
\hline \multicolumn{4}{|c|}{6 weeks after primary immunization } \\
\hline \multicolumn{4}{|c|}{ ELISA antibodies Neutralizing antibodies Lymphocyte proliferative response (SI) } \\
\hline BacSC-GP5 & $83.2 \pm 42.9$ & $11.2 \pm 4.38$ & $1.77 \pm 0.12^{\mathrm{a}}$ \\
\hline BacSC & NR & NR & $1.12 \pm 0.09^{b}$ \\
\hline PBS & NR & NR & $1.08 \pm 0.13^{b}$ \\
\hline
\end{tabular}

Data represent the mean \pm standard deviation. Different letters $(a, b, c)$ represent significant difference $(P<0.05)$ between groups; NR, no result

host.

\section{Clinical signs and histological changes after PRRSV challenge}

After PRRSV challenge, all piglets were maintained and observed for 21 days to evaluate clinical signs. Pigletsimmunized with BacSC or PBS appeared lethargic, moderate respiratory disease characterized by sneezing, coughing and markedly decreased appetite. And, the rectal temperature increased up to above $40^{\circ} \mathrm{C}$ at $4 \mathrm{dpc}$ and fluctuated greatly during challenge. Meanwhile, piglets immunized with BacSC-GP5 did not show any obvious clinical signs and the rectal temperatures were not over $40^{\circ} \mathrm{C}$ and fluctuated steady during the experiment (Figure 6). At $21 \mathrm{dpc}$, all piglets were dissected. No obvious macroscopic lesions of piglets immunized with BacSC-GP5 were found in the tonsils, spleens, kidneys and mesenteric lymph nodes except mild diffuse macular hemorrhage in the lungs. In contrast, severe hemorrhagic lungs and moderate hemorrhagic mesenteric lymph nodes were observed in piglets immunized with BacSC or PBS.

Histological changes were also evaluated in the collected lungs. Severe interstitial pneumonia was found in the lungs of piglets immunized with BacSC or PBS. In contrast, the piglets immunized with BacSC-GP5 only 


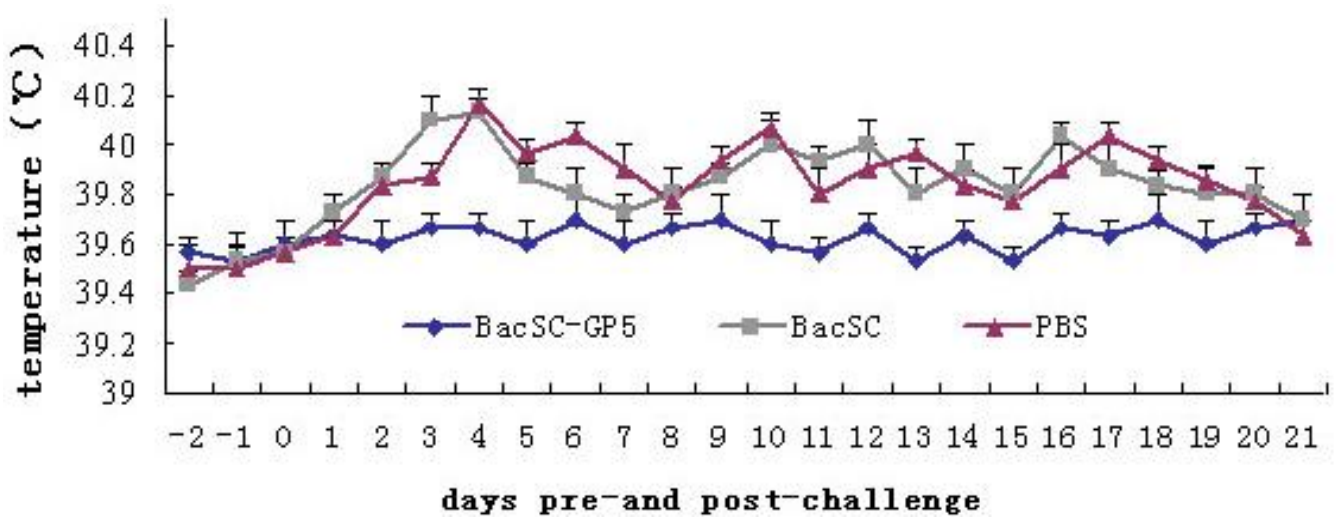

Figure 6. Mean rectal temperature of vaccinated piglets pre- and post- challenge with virulent PRRSV shaanxi strain.

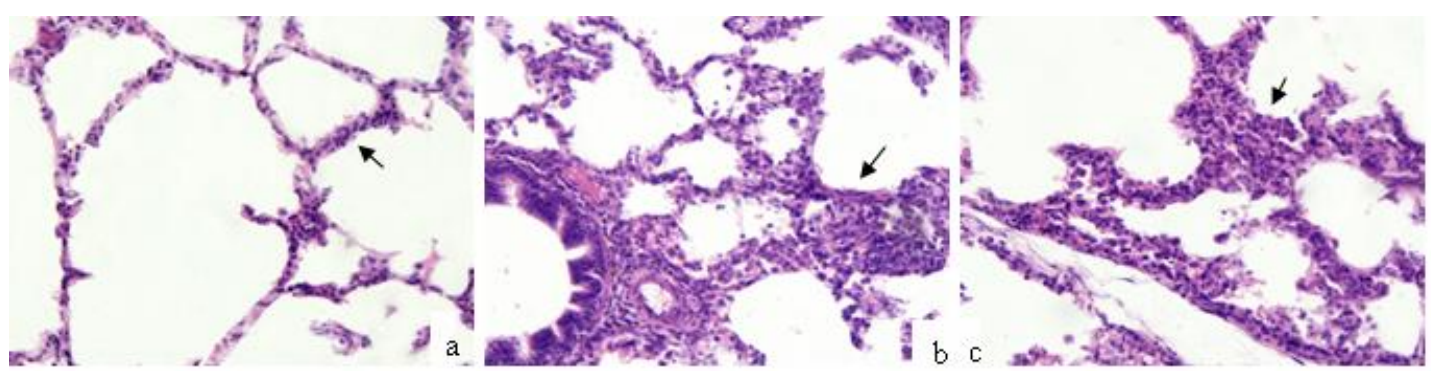

Figure 7. Histological changes in the lungs of piglets in BacSC-GP5-vaccinated group (a), BacSC group (b), and PBS group (c) at $21 \mathrm{dpc}$. a, Arrows indicate mild interstitial pneumonia with hyperemic small blood vessels; b, c Arrows indicate localized region of severe interstitial pneumonia with intensive accumulation of macrophages, and thickened alveolar septum; HE, staining. Magnification 400x.

exhibited mild interstitial pneumonia (Figure 7).

\section{Real-time RT-PCR analysis of viral replication in serum}

To determine the viral burdens in serum, the sera of five piglets in each group were collected at 7, 14 and $21 \mathrm{dpc}$ for analysis. The viral was not detected in any of the serum samples on the day of challenge. The dynamic changes of PRRSV copies for all groups are shown in Figure 8. Viruses replicated at a high level in the serum of piglets immunized with BacSC or PBS. Compared with the viral burdens from the control group, significantly reduced ones were found in the serum of piglets immunized with BacSC-GP5 at 14 and $21 \mathrm{dpc}(\mathrm{P}<0.05)$.

\section{DISCUSSION}

PRRSV infection is still a severe problem in the countries with intensive swine industries. Live attenuated and killed vaccines, the two types of commercial vaccine currently on the market, have been used wildly, and they play an extent effect in prevention and control of PRRSV. However, they cannot provide effective protection against PRRSV infection due to their inherent character (Opriessnig et al., 2002; Nilubol et al., 2004). Hence, new strategies should be developed for more efficacious vaccines against PRRSV infection. In the present study, we developed an experimental pseudotyped baculovirus vaccine and evaluated its protective efficacy. A baculovirus surface display system was firstly constructed to display the PRRSV GP5 protein as described in Figure 1. The gp64 SS would facilitate the translocation of the protein to the insect cell plasma membrane and then be cleaved, exposing the His6 tag to the outer surface, while the TM from baculovirus gp64 enabled the protein to anchor onto the plasma membrane. The CTD from baculovirus gp64 mediated the interaction with the budding nucleocapsid and the ensuing incorporation into the virus envelope (Oomens and Blissard, 1999). The results of confocal microscopy and immunogold electron microscopy indicated that the GP5 was displayed 


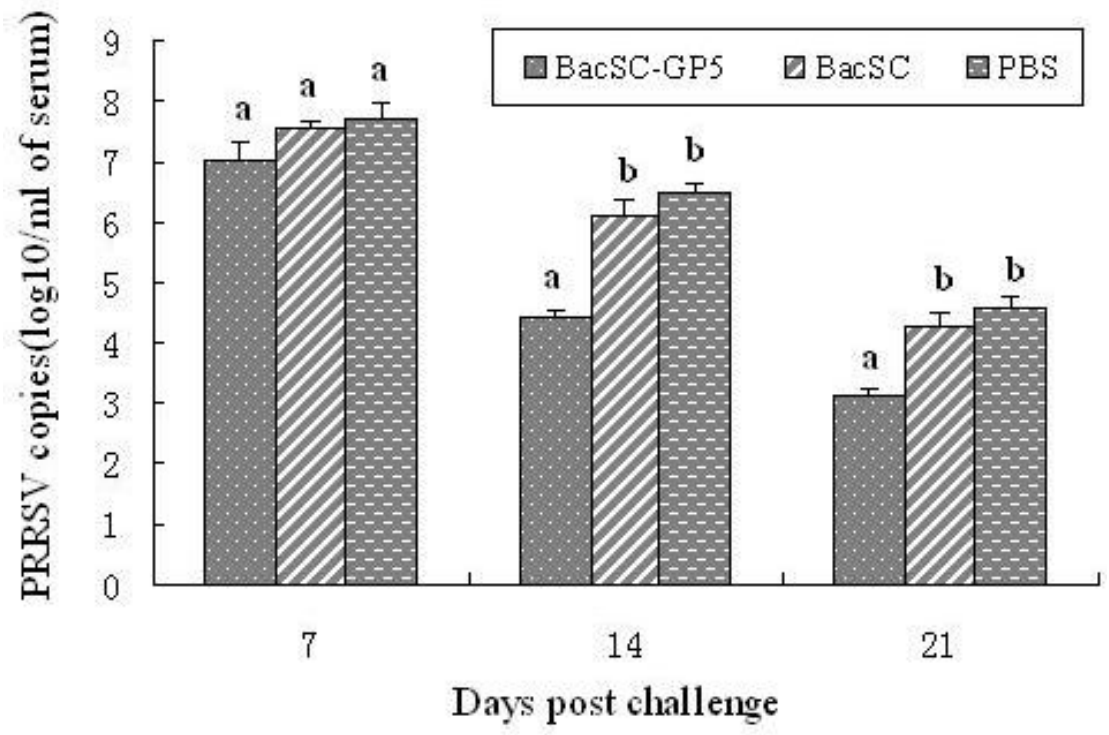

Figure 8. Real-time RT-PCR quantification of PRRSV viral copy numbers. The sera samples in each group were collected at 7,14 and $21 \mathrm{dpc}$, and the viral RNA was extracted and then reverse-transcribed to cDNA. Plasmid encoding a 308 bp fragment of PRRSV ORF7 gene was used to obtain the standard curve for quantifying the PRRSV viral genomic copy numbers. Each column represents the mean of triplicate assay with standard deviation. Different letters $(a, b, c)$ represent significant difference $(P<0.05)$ between samples.

successfully on the surface of the virus-infected cells as well as on the virions surface. The result of one-step growth curve indicated that the presence of the gp64 fusions did not alter the growth of the recombinant viruses and high titer stocks of these viruses could be achieved (Data not shown). Besides, the viral infectivity in cell cultures was also maintained.

It is now widely accepted that measures of humoral immunity can represent valuable parameters by which to evaluate the efficacy of vaccines against homologous viruses (McMurry et al., 2008). The cell-mediated immunity has also been suggested to have important implication for protective immunity against serologically distinct viruses (Doherty et al., 2006). Therefore, the simultaneous induction of strong humoral immunity and robust cellular immunity should be considered when developing a new generation of vaccines against PRRSV. In our study, we firstly evaluated the immunogenicity acquired with BacSC-GP5 in mouse model. The result showed that, significantly higher levels of GP5-specific ELISA antibody, neutralizing antibody, as well as lymphocyte proliferation responses could be induced in mice immunized with BacSC-GP5 than those received BacSC (Data not shown). Then, the immunogenicity was further evaluated in pigs. Similar results were acquired in piglets immunized with BacSC-GP5, indicating that the pseudotyped baculovirus is an alternative vector to express and present antigens of PRRSV. The strong immunogenicity of the recombinant baculovirus BacSC-GP5 may be attributable to the efficient antigen that exposes on the virions surface and increases the chance of being taken up by antigen-presenting cells (APCs). Meanwhile, protective efficacy was also evaluated by the challenge assay. The histological changes showed that piglets immunized with BacSC-GP5 exhibited mild interstitial pneumonia, while severe interstitial pneumonia was observed in those immunized with BacSC or PBS. The quantitative real-time RT-PCR also indicated that the PRRSV viral load was significantly reduced at 14 and $21 \mathrm{dpc}$ from the serum in BacSC-GP5 group than that in the control group. These data indicate that the pseudotyped baculovirus BacSC-GP5 can confer partial protection against virulent PRRSV challenge.

In our previous study, we have demonstrated that the signal peptide and hydrophobic region affect the expression of proteins in different expression vectors including prokaryotic or eukaryotic system (Wang et al., 2010). Key et al. (2001) has also demonstrated that, GP5 protein without the signal peptide region would result in a better and higher expression maintaining the same immunogenicity. In the present study, we amplified the GP5 gene without the signal peptide of $\mathrm{N}$-terminal 31 amino acid residues and demonstrated it could acquire a better immunogenicity.

It is known that a high degree of genetic and antigenic variability exists among different PRRSV isolates, which 
makes it difficult to develop efficacious PRRS vaccine (Meng, 2000). To achieve more efficient cross-protective immunity against antigenic variant, several strategies have been developed recently. For example, to express a consensus immunogenic sequence derived from different PRRSV isolates or to co-express GP5 and other immunogenic proteins of PRRSV, such as M and/or GP3, has been demonstrated to elicit a broad and crossprotective immunity (Zheng et al., 2007; Jiang et al., 2008). The baculovirus vector has the advantage of being able to accommodate a large exogenous DNA genome (>30 kb) (Hu, 2005), which makes it suitable for co-expression of multiple heterologous antigens under independent promoters and allows for simultaneous induction of high-level humoral and cellular immunity. The unique advantage of the baculovirus provides the possibility to develop more immunogenic, broader-spectrum vaccines. Presently, these studies are ongoing in our laboratory.

In conclusion, the present study describes a recombinant baculovirus BacSC-GP5 displays the GP5 protein on baculovirus. Our data demonstrate that direct vaccination with recombinant baculovirus BacSC-GP5 can develop PRRSV specific humoral and cell-mediated immune responses in natural pig host, and can confer efficient protective immunity against PRRSV. In light of our results, the baculovirus-based vaccine may hold great promise as a novel platform to prevent the PRRS epidemic and can be envisaged as an alternative option in the priming-boosting vaccination scheme.

\section{ACKNOWLEDGEMENTS}

This work was supported by Youth academic backbone support program of Northwest A and F University (No E111020901) and the New Century Excellent Talents in University of China (No.NCET-07-0701).

\begin{abstract}
Abbreviations: RT-PCR, Real-time polymerase chain reaction; ELISA, enzyme-linked immunosorbent assay; TM, transmembrane domain; CTD, cytoplasmic domain; PRRSV, porcine reproductive and respiratory syndrome virus; PRRS, porcine reproductive and respiratory syndrome; VSV-G, vesicular stomatitis virus; RNA, ribonucleic acid; PVDF, polyvinylidene fluoride; SDS-PAGE, sodium dodecyl sulfate polyacrylamide gel electrophoresis; ECL, electrochemiluminescence; PBS, phosphate-buffered saline; SI, stimulation index; $\mathbf{T C I D}_{50}$, tissue culture infection dose; dpc, days post-challenge; CPE, cytopathic effect; DMEM, Dulbecco's modified Eagle's medium
\end{abstract}

\section{REFERENCES}

Abe T, Takahashi $\mathrm{H}$, Hamazaki $\mathrm{H}$, Miyano-Kurosaki $\mathrm{N}$, Matsuura $\mathrm{Y}$, Barfoed AM, Blixenkrone-Moller M, Jensen MH, Botner A, Kamstrup S (2004). DNA vaccination of pigs with open reading frame 1-7 of PRRS virus. Vaccine, 22: 3628-3641.
Doherty PC, Turner SJ, Webby RG, Thomas PG (2006). Influenza and the challenge for immunology. Nat. Immunol., 7: 449-455.

Fan HY, Xiao SB, Tong TZ, Wang SP, Xie LL, Jiang YB, Chen HC, Fang LR (2008). Immunogenicity of porcine circovirus type 2 capsid protein targeting to different subcellular compartments. Mol. Immunol., 45: 653-660.

$\mathrm{Hu}$ YC, (2005). Baculovirus as a highly efficient expression vector in insect and mammalian cells. Acta. Pharmacol. Sinica, 26: 405-416.

$\mathrm{Hu}$ YC, Tsai CT, Chung YC, Lu JT, Hsu JTA (2003). Generation of chimeric baculovirus with histidine-tags displayed on the envelope and its purification using immobilized metal affinity chromatography. Enzyme Microb. Tech., 33: 445-452.

Jiang WM, Jiang P, Li YF, Tang JY, Wang XW, Ma S (2006a). Recombinant adenovirus expressing GP5 and M fusion proteins of porcine reproductive and respiratory syndrome virus induce both humoral and cell-mediated immune responses in mice. Vet. Immunol. Immunop., 113: 169-180.

Jiang WM, Jiang P, Wang XW, Li YF, Du YJ, Wang XL (2008). Enhanced immune responses of mice inoculated recombinant adenoviruses expressing GP5 by fusion with GP3 and/or GP4 of PRRS virus. Virus Res., 136: 50-57.

Jiang YB, Xiao SB, Fang LR, Yu XL, Song YF, Niu CS, Chen HC (2006b). DNA vaccines co-expressing GP5 and $M$ proteins of porcine reproductive and respiratory syndrome virus (PRRSV) display enhanced immunogenicity. Vaccine, 24: 2869-2879.

Jiang YB, Fang LR, Xiao SB, Zhang H, Pan YF, Luo R, Li B, Chen HC (2007). Immunogenicity and protective efficacy of recombinant pseudorabies virus expressing the two major membrane-associated proteins of porcine reproductive and respiratory syndrome virus. Vaccine, 25: 547-560.

Key KF, Haqshenas G, Guenette DK, Swenson SL, Toth TE, Meng XJ (2001). Genetic variation and phylogenetic analyses of the ORF5 gene of acute porcine reproductive and respiratory syndrome virus isolates. Vet. Microbiol., 83: 249-263.

Kitagawa Y, Tani H, Limn C, Matsunaga T, Moriishi K, Matsuura Y (2005). Ligand-directed gene targeting to mammalian cells by pseudotype baculoviruses. J. Virol., 79: 3639-3652.

McMurry JA, Johansson BE, De Groot AS (2008). A call to cellular and humoral arms: enlisting cognate $T$ cell help to develop broad-spectrum vaccines against influenza A. Hum. Vaccin., 4: 148-157.

Meng X (2000). Heterogeneity of porcine reproductive and respiratory syndrome virus: implications for current vaccine efficacy and future vaccine development. Vet. Microbiol., 74: 309-329.

Meulenberg JJ, Petersen-den Besten A, de Kluyver EP, Moormann RJ, Schaaper WM, Wensvoort G (1995). Characterization of structural proteins of Lelystad virus. Adv. Exp. Med. Biol., 380: 271-276.

Meulenberg JJM (2000). PRRSV, the virus. Vet. Res., 31: 11-21.

Nilubol D, Platt K, Halbur P, Torremorell M, Harris D (2004). The effect of a killed porcine reproductive and respiratory syndrome virus (PRRSV) vaccine treatment on virus shedding in previously PRRSV infected pigs. Vet. Microbiol., 102: 11-18.

Oomens AGP, Blissard GW (1999). Requirement for GP64 to drive efficient budding of Autographa californica multicapsid nucleopolyhedrovirus. Virology, 254: 297-314.

Opriessnig T, Halbur PG, Yoon KJ, Pogranichniy RM, Harmon KM, Evans R, Key KF, Pallares FJ, Thomas P, Meng XJ (2002). Comparison of molecular and biological characteristics of a modified live porcine reproductive and respiratory syndrome virus (PRRSV) vaccine (Ingelvac PRRS MLV), the parent strain of the vaccine (ATCC VR2332), ATCC-VR2385, and two recent field isolates of PRRSV. J. Virol., 76: 11837-11844.

Pieroni L, Maione D, La Monica N (2001). In vivo gene transfer in mouse skeletal muscle mediated by baculovirus vectors. Hum. Gene. Ther., 12: 871-881.

Qiu HJ, Tian ZJ, Tong GZ, Zhou YJ, Ni JQ, Luo YZ, Cai XH (2005). Protective immunity induced by a recombinant pseudorabies virus expressing the GP5 of porcine reproductive and respiratory syndrome virus in piglets. Vet. Immunol. Immunop., 106: 309-319.

Shen G, Jin N, Ma M, Jin K, Zheng M, Zhuang T, Lu H, Zhu G, Jin H, Jin 
M (2007). Immune responses of pigs inoculated with a recombinant fowlpox virus coexpressing GP5/GP3 of porcine reproductive and respiratory syndrome virus and swine IL-18. Vaccine, 25: 4193-4202.

Tani H, Limn CK, Yap CC, Onishi M, Nozaki M, Nishimune Y, Okahashi N, Kitagawa Y, Watanabe R, Mochizuki R, Moriishi K, Matsuura Y (2003). In vitro and in vivo gene delivery by recombinant baculoviruses. J. Virol., 77: 9799-9808.

Wang ZS, Xu XG, Tong DW, Xing FS, Chen X, Tang QL, Ning PB (2010). Cloning, Sequence Analysis and Prokaryotic Expression of ORF5 Gene of PRRSV Shaanxi Strain. Acta Agriculturae Boreali-occidentalis Sinica, 19: 1-6.

Xu XG, Liu HJ (2008). Baculovirus surface display of E2 envelope glycoprotein of classical swine fever virus and immunogenicity of the displayed proteins in a mouse model. Vaccine, 26: 5455-5460.
Yang DG, Chung YC, Lai YK, Lai CW, Liu HJ, Hu YC (2007). Avian influenza virus hemagglutinin display on baculovirus envelope: Cytoplasmic domain affects virus properties and vaccine potential. Mol. Ther., 15: 989-996.

Zheng Q, Chen D, Li P, Bi Z, Cao R, Zhou B, Chen P (2007). Co-expressing GP5 and M proteins under different promoters in recombinant modified vaccinia virus ankara (rMVA)-based vaccine vector enhanced the humoral and cellular immune responses of porcine reproductive and respiratory syndrome virus (PRRSV). Virus Genes, 35: 585-595. 